\title{
Résumé : Poids de la preuve - Facteurs à considérer lors d'une enquête sur une éclosion de maladie d'origine alimentaire
}

\author{
Vik $\mathbf{J}^{1}$, Hexemer $\mathbf{A}^{1 *}$ et Farber $\mathrm{J}^{2}$ \\ 1 Division de la gestion des éclosions, Centre des maladies infectieuses d'origine alimentaire, environnementale et zoonotique \\ de l'Agence de la santé publique du Canada, Guelph (Ontario) \\ 2 Bureau des dangers microbiens, Santé Canada, Ottawa (Ontario) \\ * Auteure-ressource : april.hexemer@phac-aspc.gc.ca
}

\section{Introduction}

Un document d'orientation, intitulé Poids de la preuve : Facteurs à considérer pour la prise de mesures appropriées et en temps opportun dans une situation d'enquête sur une éclosion de maladie d'origine alimentaire (1), a été rédigé pour aider les décideurs du gouvernement fédéral à considérer les preuves scientifiques recueillies au cours d'une enquête sur une éclosion de maladie d'origine alimentaire, et ainsi orienter les mesures d'atténuation des risques.

Le but du document est de fournir des lignes directrices sur la manière d'examiner les données probantes obtenues durant les enquêtes épidémiologiques, fournies par un laboratoire et sur la salubrité des aliments dans le contexte d'une enquête sur l'éclosion d'une maladie d'origine alimentaire, dans le cadre d'un processus global d'évaluation des risques pour la santé mené par Santé Canada. II s'agit d'un bref compte rendu du document.

\section{Approche}

Ce document a été élaboré en collaboration par Santé Canada, l'Agence de santé publique du Canada et l'Agence canadienne d'inspection des aliments, conformément à une recommandation formulée dans le rapport Weatherill, en 2009 (2). Des critères normalisés ont été établis pour apprécier les preuves épidémiologiques, fournies par un laboratoire ou issues d'une enquête sur l'innocuité des aliments, recueillies dans une situation d'enquête sur une éclosion de maladie d'origine alimentaire. Ont été décrits les facteurs à considérer, et établies des directives sur le poids à accorder aux données probantes pour chaque critère.

\section{Faits saillants}

Le Tableau 1 présente les aspects essentiels de chaque chapitre du document d'orientation relatif au poids de la preuve.

Tableau 1. Faits saillants concernant le document d'orientation relatif au poids de la preuve

\begin{tabular}{l|l}
\hline Chapitre/section & Faits saillants \\
\hline Introduction & $\begin{array}{l}\text { Une enquête sur une éclosion de maladie d'origine alimentaire est une } \\
\text { activité complexe et pluridisciplinaire qui comprend la collecte des données } \\
\text { de laboratoire, ainsi que les résultats d'enquêtes sur la salubrité des } \\
\text { aliments et d'études épidémiologiques. Au fur et à mesure que des données } \\
\text { et des renseignements détaillés sont recueillis, l'état de la situation est mise } \\
\text { à jour et, par conséquent, renforce le poids de la preuve à l'égard d'une } \\
\text { prise de mesures d'atténuation du risque. }\end{array}$ \\
\hline $\begin{array}{l}\text { Section A : Renseignements sur } \\
\text { des échantillons intacts et des } \\
\text { échantillons compromis (non } \\
\text { intacts) }\end{array}$ & $\begin{array}{l}\text { Au cours d'enquêtes sur une éclosion de maladie d'origine alimentaire, on } \\
\text { prélève souvent des échantillons d'aliments qui pourraient avoir été } \\
\text { consommés par les personnes malades. Des échantillons d'emballages } \\
\text { ouverts sont prélevés aux fins d'analyse lorsque des échantillons } \\
\text { d'emballage intact ne sont pas disponibles. Dans ces cas, une } \\
\text { contamination postérieure à l'emballage doit être envisagée. }\end{array}$ \\
\hline
\end{tabular}




\begin{tabular}{l|l}
\hline Chapitre/section & Faits saillants \\
\hline $\begin{array}{l}\text { Section B : Appariement des } \\
\text { isolats }\end{array}$ & $\begin{array}{l}\text { Des échantillons d'aliments consommés par les personnes malades sont } \\
\text { analysés pour détecter la présence de pathogènes d'origine alimentaire, } \\
\text { puis ils sont comparés avec les pathogènes préalablement isolés des } \\
\text { échantillons prélevés sur les personnes malades. Ces comparaisons entre } \\
\text { les isolats sont souvent effectuées à l'aide de techniques de typage } \\
\text { moléculaire, notamment l'électrophorèse en champ pulsé. Sont répertoriés } \\
\text { quatre critères à envisager lors de l'évaluation de la force de la preuve } \\
\text { microbiologique dans une enquête portant sur l'éclosion d'une maladie } \\
\text { d'origine alimentaire. }\end{array}$
\end{tabular}

Section C : Résumé de la preuve épidémiologique

Des preuves et des renseignements épidémiologiques directs et à l'appui sont recueillis tout au long de l'enquête sur une éclosion de maladie d'origine alimentaire. Bien que la preuve épidémiologique de référence semble découler d'une étude analytique bien conçue, il existe d'autres situations dans lesquelles le poids de la preuve serait considéré comme suffisamment fort pour justifier une mesure réglementaire fondée uniquement sur la preuve épidémiologique. Sont répertoriés neuf critères à envisager lors de l'évaluation de la force de la preuve épidémiologique dans une enquête portant sur l'éclosion d'une maladie d'origine alimentaire.

Section D : Traçabilité en amont et
en aval

Lorsqu'un aliment est mis en cause dans des cas de maladie, les enquêteurs sur la salubrité des aliments tentent de retracer l'origine de l'aliment (traçabilité en amont) et/ou les autres endroits où l'aliment a été distribué (traçabilité en aval) pour éclairer une décision de gestion du risque. Cinq situations sont présentées pour être utilisées à titre de guide afin d'obtenir le poids de la preuve nécessaire pour diffuser un rappel et/ou prendre toute autre mesure de gestion du risque dans le but de veiller à l'identification de tout produit contaminé et de découvrir la source de contamination.

\author{
Section E : Évaluation du risque \\ pour la santé
}

Les évaluateurs techniques de l'Agence canadienne d'inspection des

\begin{tabular}{l|l}
\hline $\begin{array}{l}\text { Section F : Définitions des risques } \\
\text { pour la santé }\end{array}$ & $\begin{array}{l}\text { Le niveau du risque pour la santé est déterminé par la prise en compte de la } \\
\text { détermination des dangers, de l'évaluation de l'exposition et de la } \\
\text { caractérisation du danger. Les définitions des trois catégories de risque pour } \\
\text { la santé sont fournies. }\end{array}$ \\
\hline $\begin{array}{l}\text { Section G : Mesures de gestion du } \\
\text { risque possibles à la suite d'une } \\
\text { évaluation de risque pour la santé }\end{array}$ & $\begin{array}{l}\text { À la suite d'une évaluation de risque pour la santé, un certain nombre de } \\
\text { mesures de gestion des risques peuvent être prises. Le type de mesures } \\
\text { prises varie en fonction du niveau du risque pour la santé et d'autres } \\
\text { facteurs. }\end{array}$ \\
\hline $\begin{array}{l}\text { Section H : Exemples de mises en } \\
\text { situation }\end{array}$ & $\begin{array}{l}\text { Les études de cas sont présentées afin de démontrer comment le poids de } \\
\text { la preuve est envisagé en vue d'une intervention dans le contexte d'une } \\
\text { enquête sur l'éclosion de maladie d'origine alimentaire. Ces études de cas } \\
\text { sont présentées à des fins d'orientation seulement. }\end{array}$ \\
\hline
\end{tabular}
aliments et/ou les provinces et territoires demandent des évaluations des risques pour la santé en ce qui concerne les dangers microbiologiques. Celles-ci sont effectuées par Santé Canada pour des situations d'éclosions de maladie d'origine alimentaire. Une équipe d'évaluation scientifique y évalue les données recueillies durant les enquêtes de laboratoire, les enquêtes sur la salubrité des aliments et les enquêtes épidémiologiques, puis attribue un niveau de risque pour la santé en fonction des informations disponibles au moment de la demande d'évaluation des risques.

Le niveau du risque pour la santé est déterminé par la prise en compte de la détermination des dangers, de l'évaluation de l'exposition et de la caractérisation du danger. Les définitions des trois catégories de risque pour la santé sont fournies.

A la suite d'une évaluation de risque pour la santé, un mesures de gestion des risques peuvent être prises. Le type de mesures prises varie en fonction du niveau du risque pour la santé et d'autres urs.

la preuve est envisagé en vue d'une intervention dans le contexte d'une sont présentées à des fins d'orientation seulement. 


\section{Résultats}

L'ensemble des preuves épidémiologiques, fournies par un laboratoire et issues d'une enquête sur l'innocuité des aliments est examiné au moyen d'une évaluation des risques pour la santé, puis un niveau de risque pour la santé est attribué à l'aliment en cause. Le document d'orientation portant sur le poids de la preuve a joué un rôle central dans la conduite des évaluations des risques pour la santé, ce qui a facilité l'adoption de mesures d'atténuation des risques opportunes et appropriées. Le document est actuellement soumis à une révision menée par Santé Canada, laquelle devrait être achevée en 2014.

\section{Conclusion}

L'utilisation d'un document d'orientation proposant des critères normalisés pour évaluer le poids de la preuve dans une situation d'enquête sur une éclosion de maladie d'origine alimentaire facilite l'achèvement en temps opportun des évaluations des risques pour la santé des vecteurs alimentaires suspects et permet d'orienter la mise en œuvre d'interventions de santé publique visant à atténuer les risques d'origine alimentaire pour les consommateurs.

\section{Conflit d'intérêts}

Aucun conflit d'intérêts à déclarer.

\section{Financement}

Aucun financement externe n'a été reçu.

\section{Références}

(54) Gouvernement du Canada. Poids de la preuve : Facteurs à considérer pour la prise de mesures appropriées et en temps opportun dans une situation d'enquête sur une éclosion de maladie d'origine alimentaire. Janvier 2011. http://www.hc-sc.gc.ca/fn-an/pubs/securit/2011-food-illness-outbreak-eclosion-malad-ailments/index-fra.php

(55) Gouvernement du Canada. Rapport Final - Enquête relative à la listériose de 2008. Juillet 2009. http://www.cpha.ca/uploads/history/achievements/09-lirs-rpt f.pdf 